\title{
The correlation between wing kinematics and steering muscle activity in the blowfly Calliphora vicina
}

\author{
Claire N. Balint* and Michael H. Dickinson \\ Department of Integrative Biology, University of California, Berkeley, CA 94720, USA \\ *e-mail: cnbalint@socrates.berkeley.edu
}

Accepted 5 October 2001

\begin{abstract}
Summary
Determining how the motor patterns of the nervous system are converted into the mechanical and behavioral output of the body is a central goal in the study of locomotion. In the case of dipteran flight, a population of small steering muscles controls many of the subtle changes in wing kinematics that allow flies to maneuver rapidly. We filmed the wing motion of tethered Calliphora vicina at high speed and simultaneously recorded multi-channel electromyographic signals from some of the prominent steering muscles in order to correlate kinematics with muscle activity. Using this analysis, we found that the timing of each spike in the basalare muscles was strongly

to each muscle spike decreased with increasing levels of stroke deviation. This result suggests that downstroke deviation is controlled in part via the mechanical summation of basalare activity. We also found that interactions among the basalares and muscles III2-III4 determine the maximum forward amplitude of the wingstroke. In addition, activity in muscle I1 appears to participate in a wingbeat gearing mechanism, as previously proposed. Using these results, we have been able to correlate changes in wing kinematics with alteration in the spike rate, firing phase and combinatorial activity of identified steering muscles.
\end{abstract} correlated with changes in the deviation of the stroke plane during the downstroke. The relationship was nonlinear such that the magnitude of the kinematic response
Key words: insect flight, blowfly, Calliphora vicina, motor output, kinematics, steering.

\section{Introduction}

The musculoskeletal system is an ultimate downstream 'decoder' of neural signals, converting the activity of motor neurons into the kinematics we observe as the behavior of an organism. Functioning at the interface between the nervous system and the external world, muscles act as neural output transducers, reversely analogous to sense organs that act as stimulus input transducers (Young, 1989). In sensory systems, it has been recognized that incoming information must be encoded in real time and that, for time-varying stimuli, the nervous system does not have the luxury of signal-averaging. For example, mathematical real-time decoding methods have revealed the relatively large information content encoded by a small number of spikes in single sensory neurons (for a review, see Rieke et al., 1997). Similarly, in motor systems, ongoing adjustments must be made on a moment-by-moment basis, but the various components of motor pattern conversion, especially the intervening muscles and morphological structures, ensure that the process will be very non-linear (Dickinson et al., 2000; Hooper and Weaver, 2000). In the case of insect flight, the neuro-mechanical conversion is a multi-step process whereby a pattern of activation within a motor neuron leads to twitches in the muscle it innervates, the force from the twitches moves or deforms the sclerite to which the muscle is attached, the change in the position of the sclerite modifies the motion of the wing and the change in wing motion leads to an alteration in aerodynamic force.

Several studies have addressed aspects of motor encoding in insect flight by quantifying the muscle spiking patterns, largely in locusts (Möhl, 1985; Möhl and Zarnack, 1977; Pearson and Wolf, 1987; Schmidt and Zarnack, 1987; Wendler, 1974; Wilson and Weis-Fogh, 1962; Zarnack and Möhl, 1977), moths (Kammer, 1971; Wendler et al., 1993) and flies (Egelhaaf, 1989; Götz, 1983; Heide, 1983; Nachtigall and Wilson, 1967). Further, the coupling process linking changes in muscle activity to adjustments in wing kinematics has been studied through correlational studies in locusts and flies: Locusta (Dawson et al., 1997; Thuring, 1986; Zarnack, 1988); Schistocerca (Fischer and Kutsch, 1999; Waldman and Zarnack, 1988); Drosophila (Heide and Götz, 1996; Lehmann, 1994; Lehmann and Götz, 1996); Calliphora (Heide, 1983; Tu and Dickinson, 1996). However, given the potential non-linearities of muscle mechanics, the unknown nature of the coupling between muscles, joint morphology and the wing, as well as the highly variable motor responses to open-loop sensory conditions, our understanding of the transfer functions between motor patterns and wing movement is quite simplistic. In addition, information regarding the full extent of variation in wing kinematics has lagged behind the analysis of firing patterns in flight muscles. 
Although flies are among the most aerodynamically sophisticated fliers, certain features of their flight control system are relatively simple and amenable to experimental studies. Flies use a single pair of wings and the muscles are segregated into distinct functional groups (for a review, see Dickinson and Tu, 1997). Many of the subtle alterations in wing kinematics that allow flies to execute fast changes in flight trajectory are accomplished by the action of small steering muscles that insert directly onto the sclerites at the base of the wing. The differential responses of several of these steering muscles to the direction (Heide, 1968, 1971b, 1975, 1983; Heide and Götz, 1996) and time course (Egelhaaf, 1989) of visual stimuli have corroborated their presumed role in mediating certain flight behaviors. The 13 steering muscles are attached to four sclerites at the wing hinge: the basalar sclerite, the pterales I and III and the posterior notal wing process. Five additional synchronous muscles thought to be involved in flight control are not directly attached to the sclerites of the wing hinge.

Inferences from the morphology of the hinge and its muscle attachments, together with the tonic firing patterns of their muscles, have given rise to several models of steering muscle function (Dickinson and Tu, 1997; Heide, 1968, 1971a,b; Miyan and Ewing, 1985; Nachtigall and Wilson, 1967; Wisser, 1987; Wisser and Nachtigall, 1984). Because of their similar response properties, Heide $(1975,1983)$ proposed synergistic effects among muscles. Specifically, the second muscle of the basalare (b2) and the first muscle of pterale III (III1) act together to increase stroke amplitude, whereas the first muscle of pterale I (I1) and the third muscle of the posterior notal wing process (hg3) act to decrease stroke amplitude. In the fruitfly Drosophila melanogaster, the bursting activity in various individually recorded steering muscles is correlated with changes in stroke amplitude over many wingbeat cycles (Heide and Götz, 1996). In the blowfly Calliphora vicina, the timing of individual basalare muscle spikes within a single wingbeat cycle can have clear cycle-bycycle effects on wingbeat kinematics (Tu and Dickinson, 1996). Lehmann and Götz (1996) confirmed the influence of firing phase on stroke kinematics by stimulating b2 at various points within the stroke cycle. In addition to the effects of individual muscles on wingbeat kinematics, the analysis of $\mathrm{Tu}$ and Dickinson (1996) also suggested the existence of functional synergies within the population of steering muscles. However, these muscles have not previously been recorded in concert, nor in combination with high-speed video.

To quantify the contribution of multiple muscle groups to kinematic variation, we extend the methodology of Tu and Dickinson (1996) to include multiple, simultaneous steering muscle recordings combined with high-speed videography. We correlate kinematic changes with the temporal and spatial activity patterns of steering muscles on a cycle-by-cycle basis. The results extend the work of previous studies and provide some new hypotheses concerning the processes linking motor activity and flight behavior.

\section{Materials and methods \\ Insects}

Male blowflies (Calliphora vicina R.-D.) were selected from a laboratory colony, maintained at approximately $22^{\circ} \mathrm{C}$ with a $12 \mathrm{~h}: 12 \mathrm{~h}$ light:dark cycle. All individuals were between 1 and 2 weeks post-eclosion at the time of tethering. Wing lengths (measured from basicosta to wingtip) ranged from 8.9 to $9.7 \mathrm{~mm}$ post-mortem.

\section{Tethering procedure}

We anesthetized each fly by placing it in a freezer at $-4{ }^{\circ} \mathrm{C}$ for 3-4 min. The fly was then immediately tethered at the dorsal midline of the mesopraescutum to a modified no. 0 insect pin as described by Tu and Dickinson (1996). As a modification to the earlier procedure, we soldered short lengths of $0.025 \mathrm{~mm}$ diameter nickel chromium (NiChr) wire with formvar insulation (A-M Systems) to the terminals of five pairs of 28 gauge wires glued to the tether. We implanted the tips of a pair of the $\mathrm{NiChr}$ wires into each of 4-5 steering muscles on the left side of the animal through holes in the cuticle made with an etched tungsten needle. In all nine animals used in the electrophysiological analysis, paired electrodes were implanted into muscles b2, b1, III1 and I1. In six of these animals, electrodes were also implanted in the vicinity of muscles III2-III4. Correct implantation of each pair of electrodes was verified by qualitative differences in firing rate (e.g. high rates in b1 relative to b2) and the extent of cross-talk with nearby muscles (e.g. I1 recordings include cross-talk from b2, while III1 recordings include cross-talk from I1 but not from b2).

To create markers for digitization, we used a sharpened wooden applicator stick to place a small dot of silver (Palmer Prism Acrylic) or iridescent pearl (Golden Acrylics) paint on the basicosta (wing base) and at the distal tip of the wing posterior to the junction of vein $\mathrm{R}_{4+5}$. Each fly was allowed to recover for 1 day following initial electrode implantation before filming began, but we often made adjustments in electrode placement between filming sequences. Data were collected for 2-4 consecutive days following initial electrode implantation. When flies were not being used in experiments, they were suspended over a polypropylene ball floating in a beaker of water. The flies drank water ad libitum from the ball and were fed sugar water twice daily via a hand-held syringe.

\section{Filming}

Flies were kept under fully lit laboratory conditions (500 lx) starting at least $1 \mathrm{~h}$ prior to and during filming. We secured the free end of the tether onto a piezoelectric crystal attached to a rigid acrylic rod. The end of the rod was aligned using an adjustable manipulator, so that the fly's longitudinal body axis was between $0^{\circ}$ and $15^{\circ}$ relative to horizontal and its sagittal plane was parallel to the camera's focal plane. The video camera lens was $42-45 \mathrm{~cm}$ from the midline of the fly. A polypropylene ball floating within a beaker of water was placed under the fly to allow it to stand or walk between flight bouts.

We filmed the flies at a rate of 3000 frames s$^{-1}$ using a Kodak Ektapro equipped with an Intensified Imager and a $90 \mathrm{~mm}$ 


\section{Wing kinematics and steering muscle activity in Calliphora vicina 4215}

A

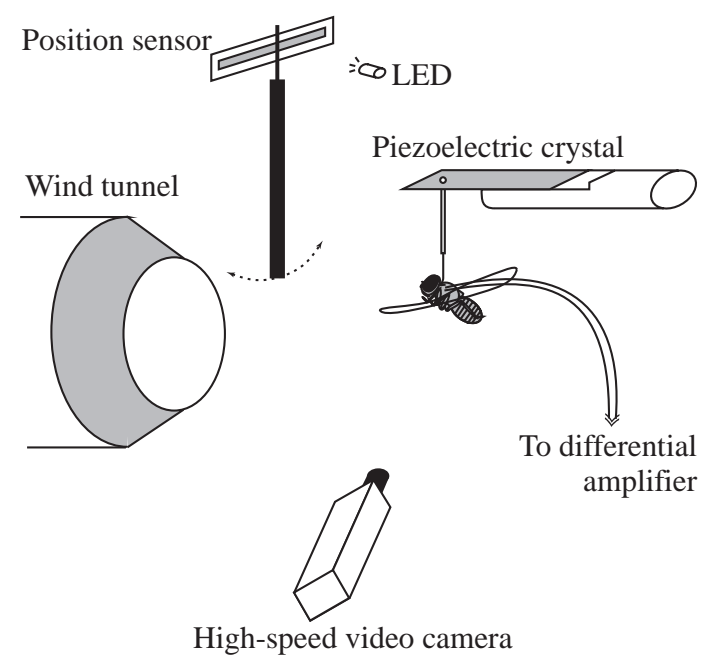

B
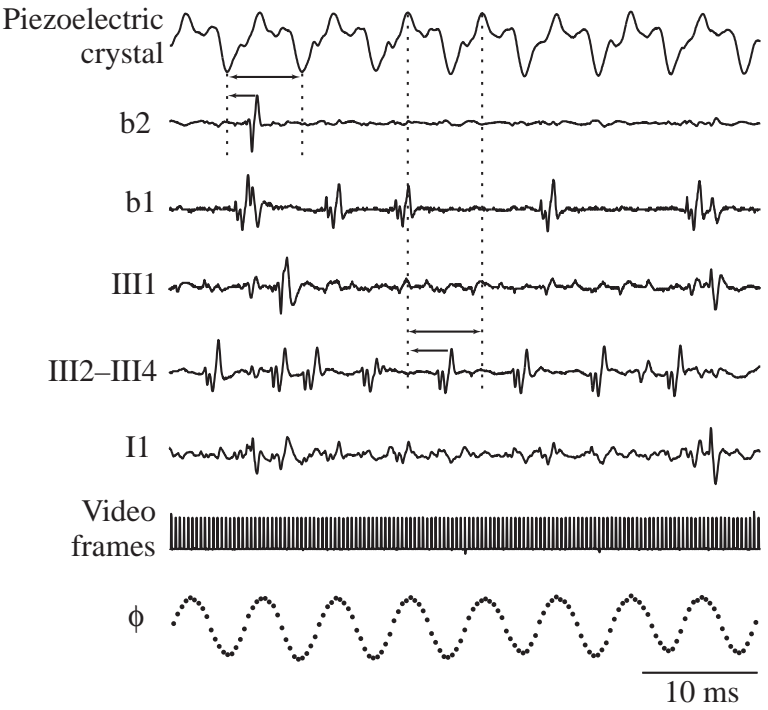

Fig. 1. Schematic cartoon showing the method of data collection (not drawn to scale). (A) Tethered flies were suspended beneath a piezoelectric crystal in front of a wind tunnel with their left side facing the high-speed video camera. A brass rod was suspended with its base between the fly's head and the wind tunnel and its upper end between an LED and a position sensor. Four to five pairs of electrode wires were implanted into the left side of the fly's thorax. (B) Sample simultaneous data traces from five EMG channels, the piezoelectric crystal and framemarks from the high-speed video camera, shown with the kinematic wing elevation angles $(\phi)$ digitized and calculated from the concurrent video frames. The phase of each muscle spike was quantified relative to the piezoelectric oscillations as described in the text (see arrows and broken vertical lines).

macro lens (Tamron). The video camera was tilted $30^{\circ}$ relative to the ground to capture the wingbeat trajectory optimally within the $192 \times 80$ pixel field of view. Extracellular potentials from the implanted electrodes were amplified using an a.c. amplifier (A-M Systems; model 1800) and digitized using a Digidata 1200 and Axoscope software (Axon Instruments). Wingbeat signals from the piezoelectric crystal and frame-mark signals from the EktaPro Hi-Spec Processor were also recorded. Acquisition rates were 29, 37, or $42 \mathrm{kHz}$ per channel depending on the number of input signals. All three types of signal, the wingbeat signals, the electromyographic (EMG) signals and the framemark signals, were displayed along a common time base to allow for fine temporal correlation among channels.

Wind and visual stimuli were presented to the fly as described previously (Tu and Dickinson, 1996). The mouth of a small open-throated wind tunnel was positioned directly in front of the fly, approximately $5 \mathrm{~cm}$ from the front of its head. A $7.0 \mathrm{~cm} \times 0.8 \mathrm{~cm}$ black cylindrical brass rod was suspended between the fly and the wind tunnel, with the base of the rod level with the fly's head (Fig. 1). This pendulum rod oscillated at a frequency of approximately $1 \mathrm{~Hz}$. We increased the contrast between the black rod and the background by fitting the top of the wind tunnel with a white cardboard panel. In a few cases, the position of the pendulum was monitored by placing the rod's upper end between a light-emitting diode (LED) and an optoelectronic position sensor (UDT). In these cases, the output of the light sensor was recorded concurrently with the other signals.

The thorax and near (left) wing of the fly were illuminated using two fiber-optic light sources (Fostec). A pair of fiber-optic arms illuminated the fly from above, the other pair from below, and all light sources were directed away from the eyes. As the polypropylene ball was lowered away from the fly, the wind tunnel was switched on and the pendulum rod was set into motion. If and when the fly reacted to the pendulum with periodic head movements, wingbeat amplitude modulations and spike bursting in b2, a manually activated external trigger initiated video capture and electrophysiological data acquisition. Each video sequence comprised 6552 frames and lasted approximately $2.2 \mathrm{~s}$.

Data were collected from a total of 11 animals. Five animals with implanted electrodes were also filmed without the visual stimulus. Two animals were filmed prior to and following electrode implantation, and two animals were filmed solely without implanted electrodes.

\section{Kinematic data analysis}

All recorded video images were transferred from the EktaPro Hi-Spec Processor onto VHS videotape, and selected sequences were downloaded onto computer. For each sequence analyzed, we used Scion Image software to digitize the wingtip once per frame and the basicosta once per sequence. We manually digitized wingtip coordinates from a total of 144150 frames taken from 21 selected video sequences of 11 flies.

While it is most convenient to quantify wing motion relative to the stroke plane, we found that in Calliphora vicina the mean stroke plane varies from stroke to stroke and thus cannot serve as a straightforward reference frame. Therefore, we defined the wingtip coordinates morphologically relative to the 
body to facilitate comparisons within sequences and among individuals. First, the $X$ and $Y$ coordinates of the basicosta $\left(x_{0}, y_{0}\right)$ were subtracted from each wingtip coordinate so that the basicosta acted as the origin. Second, the body axis was defined as the line connecting the top of the calipter and the mid-posterior border of the eye. We defined the inclination angle of the body $(\chi)$ as the angle between this line and the horizontal axis of the video screen. To combine ease of interanimal comparison with that of aerodynamic interpretation, we rotated the video screen coordinate system so that the inclination of the body was at $35^{\circ}$ relative to horizontal:

$$
\begin{aligned}
& x^{\prime}=\left(x-x_{0}\right) \cos \left(35^{\circ}-\chi\right)+\left(y-y_{0}\right) \sin \left(35^{\circ}-\chi\right), \\
& y^{\prime}=\left(y-y_{0}\right) \cos \left(35^{\circ}-\chi\right)+\left(x-x_{0}\right) \sin \left(35^{\circ}-\chi\right) .
\end{aligned}
$$

With these transformations, $x^{\prime}$ and $y^{\prime}$ measured motion about an inclined body axis. This transformation resulted in a roughly horizontal average stroke plane for the sequences within our analysis.

The two-dimensional Cartesian coordinates of the wingtips were then converted to polar coordinates. The $z$ coordinate was calculated assuming a constant wing length:

$$
z^{\prime}=\left(L^{2}-x^{\prime 2}-y^{\prime 2}\right)^{1 / 2}
$$

where $L$ is the length of the wing in the coordinate system of the digitized image, and $z^{\prime}$ is the coordinate in the plane perpendicular to the $x^{\prime}, y^{\prime}$ plane. In previous descriptions of wing motion during flight, wing flexion appears to be minimal, except for a slight bending at the end of the downstroke (Nachtigall, 1966; Wootton, 1981). At present, the extent to which wing length stays constant has not been quantitatively measured. The polar coordinates, deviation $(\theta)$ and elevation $(\phi)$ angle, of each wingtip coordinate were calculated relative to this new coordinate system (Fig. 2):

and

$$
\phi=\sin ^{-1}\left[x^{\prime}\left(x^{2}+z^{2}\right)^{1 / 2}\right]
$$

$$
\theta=\sin ^{-1}\left(y^{\prime} / L\right)
$$

\section{Electrophysiological data analysis}

We sorted spikes recorded from each EMG channel on the basis of threshold and shape using a custom-designed program written in MatLab (Mathworks). Although most muscle recordings were contaminated by cross-talk, its source was easily recognized by careful comparison with other channels. For muscles b2, b1 and I1, we quantified the firing phase $(\psi)$ of each spike relative to the minima in piezoelectric oscillations:

$$
\psi=\left(\frac{S-m_{1}}{m_{2}-m_{1}}\right) 100,
$$

where $S$ is the time of occurrence of the largest maximum or minimum in the muscle spike waveform, $m_{1}$ is the time of the piezoelectric minimum preceding the spike and $m_{2}$ is the time of the minimum following the spike. Correlation with digitized kinematics from high-speed video recordings indicated that the

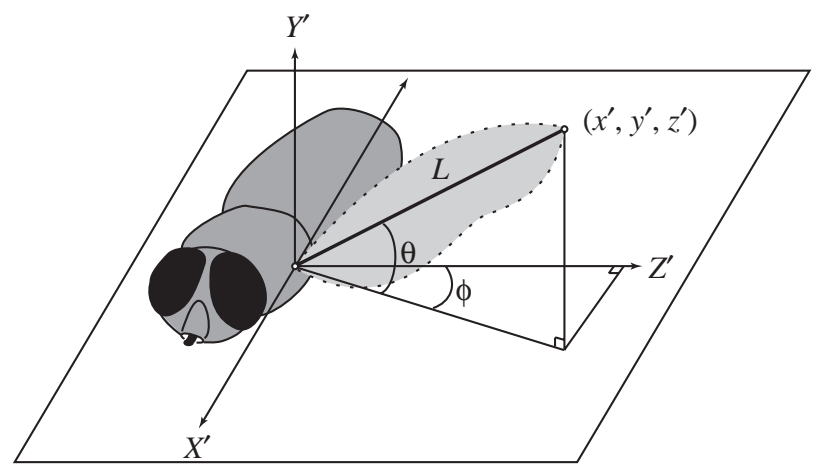

Fig. 2. Coordinate system for measuring wing kinematics. The polar coordinate angles, deviation $(\theta)$ and elevation $(\phi)$, of the wing in each frame were measured relative to the rotated body axis as described in the text.

minima in the piezoelectric record corresponded roughly to the minima of wing elevation (Fig. 1). For muscles III1 and III2-III4, we quantified phase in the same manner relative to the maxima in piezoelectric oscillations. Assuming that our piezoelectric signal is a good approximation of oscillations in muscle length and that the delays between the spike in a muscle and its subsequent activation are constant, our method of phase calculation provides an estimate of the relative phase of muscle activation.

\section{Results \\ Firing characteristics}

During the visually induced oscillatory turning reactions in this study, all steering muscles tended to fire in bursts driven by the visual stimulus (Fig. 3A,B). The variation in firing rates in these simultaneous recordings was generally consistent with the pattern reconstructed by Heide (1983) from individually recorded muscles. Muscles b1, b2, III1 and III2-III4 fired maximally when the pendulum was contralateral to the recording site, roughly correlated with an increase in wing stroke amplitude, while I1 fired maximally when the pendulum was ipsilateral, correlated with a decrease in stroke amplitude. The maximum sustained spike rate was one spike per wingbeat cycle in all muscles (see Kinematic characteristics below for observed wingbeat frequencies), although we occasionally observed two spikes in a single cycle, most often in III2-III4.

Muscles b2 and III1 displayed similar activity patterns and tended to fire in relatively short bursts, which began with a rapid increase in firing frequency, followed by a steady decrease (Fig. 3B). Muscle I1, although active antiphasically with b2 and III1, also fired in short bursts. In contrast, b1 and III2-III4 tended to sustain their maximal firing frequency for longer portions of each flight sequence.

Summed over all recorded flight sequences, each muscle displayed a variety of firing phases, distributed around a characteristic preferred phase band within the wingbeat cycle (Fig. 4A). The preferred phase bands for each muscle were consistent with those observed by Heide (1983) from 
A

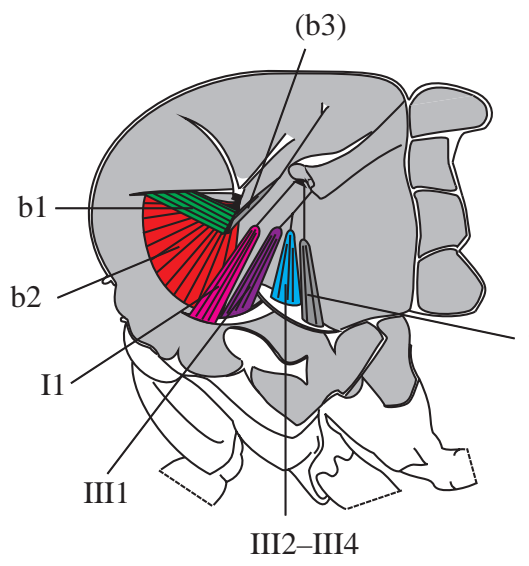

Anterior $\longleftarrow$

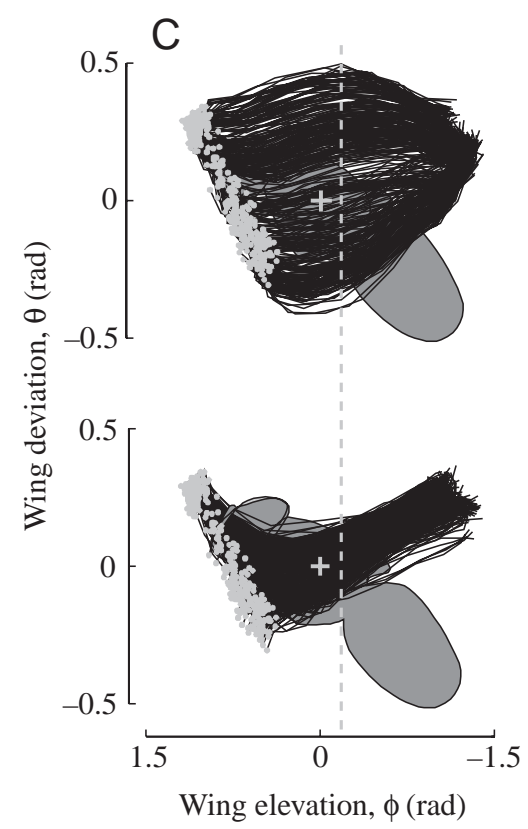

B<smiles>[20BH]</smiles>

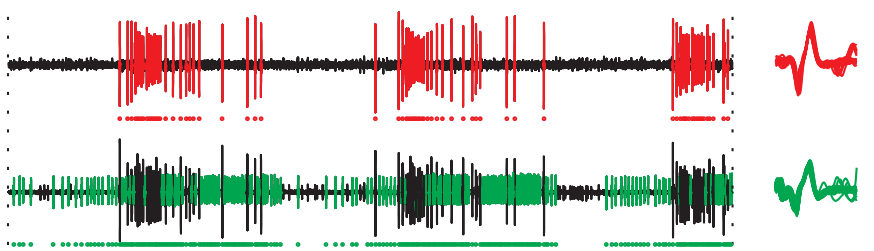

III1

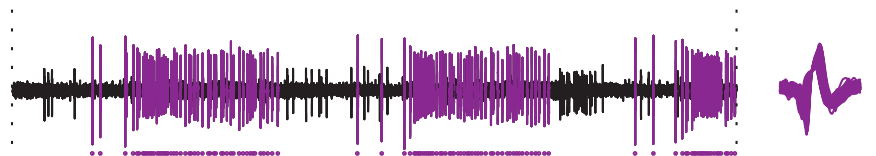

III2-III4
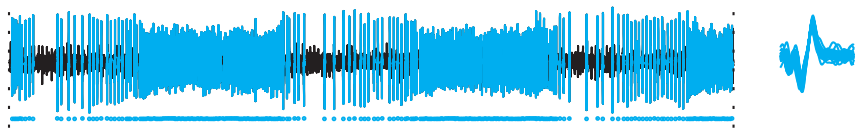

I1
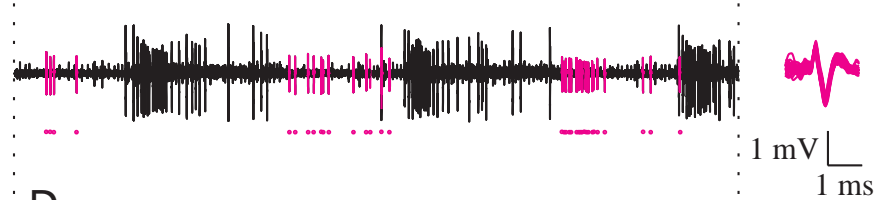

D
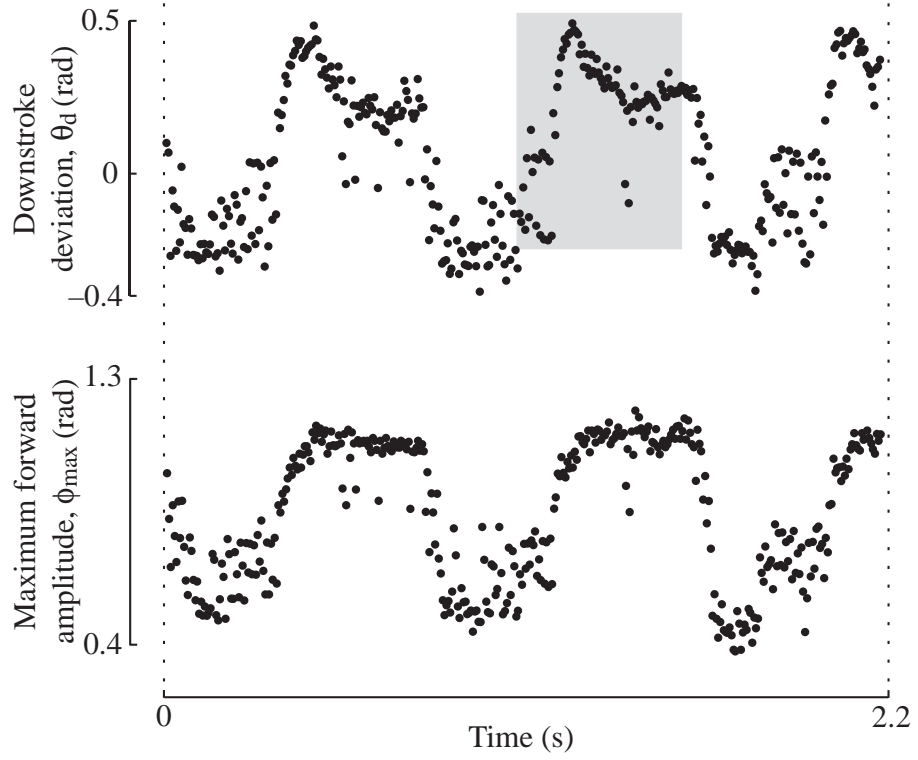

Fig. 3. Simultaneous steering muscle EMG recordings and kinematic measurements. (A) Diagram of the anatomy of the direct steering muscles recorded in this study. Two additional muscles attached at common sclerites are shown and marked by parentheses. (B) Sample EMG recording during one sequence of visually induced oscillatory turning. Each spike occurrence is reproduced as a raster of dots below each EMG trace. Each spike has been color-coded and is shown overlain on an expanded time base at the right. (C) Total set of wingbeat trajectories filmed simultaneously with EMG recordings in B separated into downstrokes (above) and upstrokes (below). The cross marks the wing base, which served as the origin. The dashed line indicates a wing elevation of $-0.2 \mathrm{rad}$. Downstroke deviation was defined as the wing deviation at the point of intersection of the downstroke trajectory with the dashed line. Stroke amplitude was defined as the maximum anterior wing elevation in each cycle, marked by gray circles. (D) Downstroke deviation and maximum forward amplitude of each cycle plotted as a function of time. Plots in D and B share the same time scale. The gray box in the deviation plot in D indicates the section shown in more detail in Fig. 6.

individually recorded muscles. Muscles b1 and b2 fired with highest probability near the time of pronation, I1 tended to fire during the downstroke and III1 and III2-III4 tended to fire near the time of supination. Within flight sequences, the time course of firing phase variation also differed among muscles (Fig. 4B). The most tonically active muscles, b1 and III2-III4, displayed gradual shifts in spike phase $(\psi)$ within a firing burst. The occasional shifts in the firing phases of b2 and I1 were sporadic, and the firing phase of III1 varied widely from spike to spike.

\section{Kinematic characteristics}

Within all sets of wingtip trajectories, we noted the most variation in wing deviations during the downstroke and the maximum anterior extent of the wing at ventral reversal 

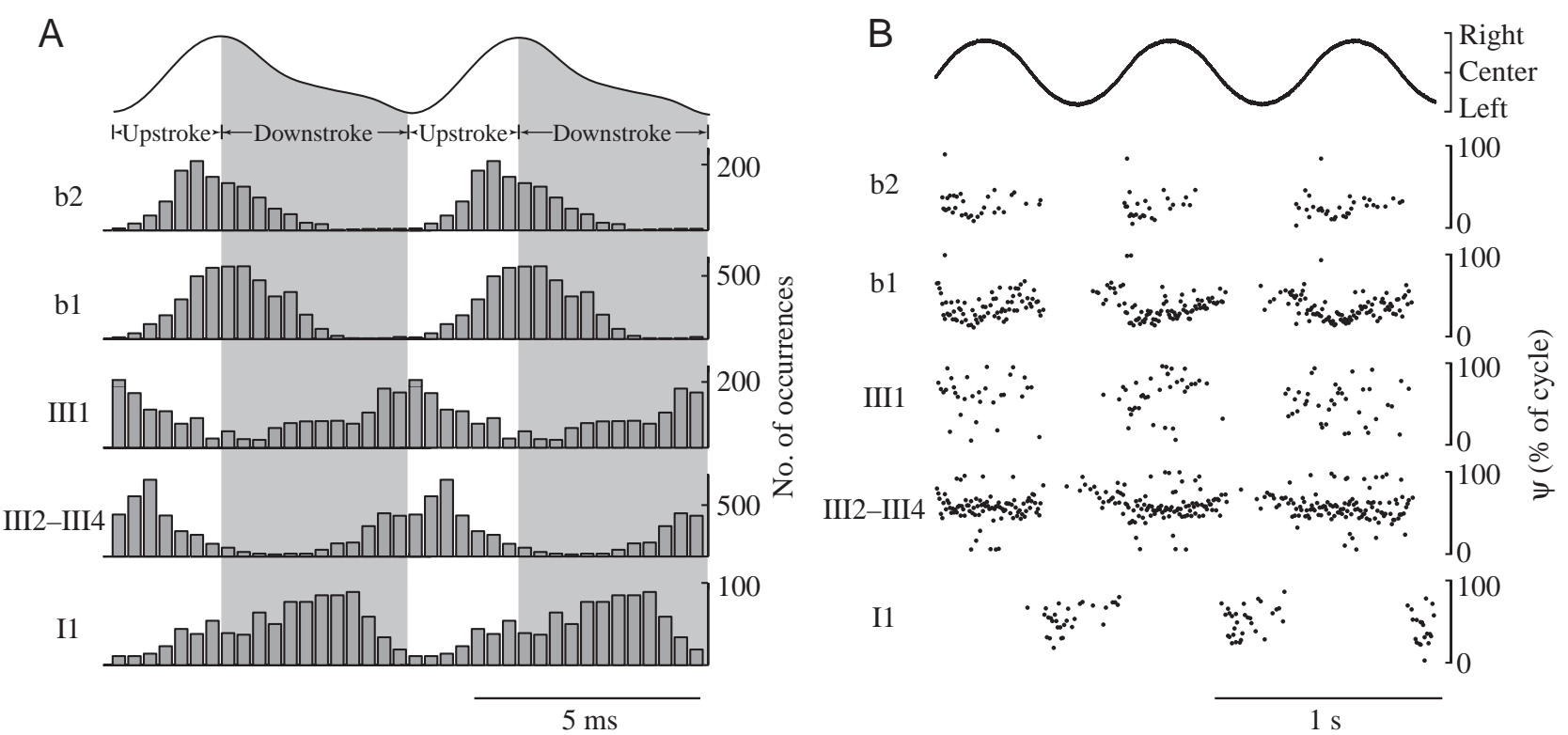

Fig. 4. Observed spike phases. (A) Composite phase histogram of all muscle spikes, shown relative to the wingbeat cycle. A single histogram is repeated twice to emphasize the cyclic nature of the firing pattern. Spike counts for muscles b2, b1, III1 and I1 came from 21 sequences recorded from nine individuals. Spike counts for III2-III4 came from 15 sequences recorded from five individuals. (B) Sample plot of phase $(\psi)$ variation seen in a single sequence over time. The top trace represents the position of the pendulum during the sequence.

(Fig. 3C). To simplify the analysis of kinematic data, we obtained one value per wingbeat cycle for each of these two kinematic features within the polar coordinate system defined in equations 3 and 4 . We defined downstroke deviation, $\theta_{\mathrm{d}}$, as the wing deviation angle, $\theta$, during the downstroke as the wing crosses a plane fixed at $\phi$ equal to $-0.2 \mathrm{rad}$. Maximum forward amplitude, $\phi_{\max }$, was the maximum value of $\phi$ in each cycle. When plotted throughout the flight sequence, both kinematic variables increased and decreased in time with the visual stimulus and with the bursts of muscle spikes, but they differed somewhat in their time course (Fig. 3D). Downstroke deviation tended to vary more from one cycle to the next, whereas the maximum stroke amplitude was more stable, especially at high values. Individuals without implanted electrodes displayed the same basic pattern of kinematic changes (Fig. 5), indicating that these patterns were not artefacts of electrode implantation. Wingbeat frequencies varied subtly within and among flight sequences as measured by peak-to-peak wavelengths of the piezoelectric signal $(164.1 \pm 10.5 \mathrm{~Hz}$, mean \pm S.D., $N=3897$ cycles from 11 individuals).

\section{Downstroke deviation and spike occurrence}

There are at least three features of steering muscle activity that might contribute to the control of wingbeat kinematics: spike rate, spike phase and combinatorial effects among different steering muscles. Because of the strong correlations found between basalare muscle activity and cycle-by-cycle adjustments of wingbeat kinematics in an earlier study (Tu and Dickinson, 1996), we first examined the influence of the basalare muscles b1 and b2. Muscles b1 and b2 generally fired at most one spike per wingbeat cycle, so we classified each cycle into three categories: cycles during which both b1 and b2 fired, those during which only b1 fired and those during which neither b1 nor b2 fired. A fourth category was not required because spikes in b2 rarely occurred within a wingbeat cycle without the occurrence of a b1 spike in the same cycle. When b1 and b2 fired at a low frequency, a spike in either muscle was associated with a transient increase in $\theta_{d}$ (Fig. 6A). When the frequency of b2 increased, $\theta_{\mathrm{d}}$ first rose to a peak and later declined to a plateau as $\mathrm{b} 2$ frequency decreased
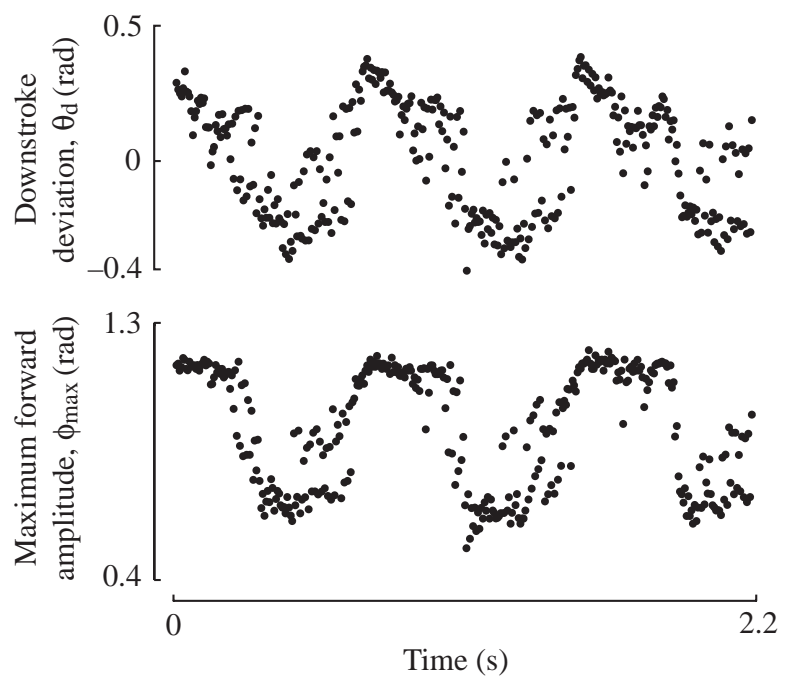

Fig. 5. Downstroke deviation and maximum forward amplitude plots from one flight sequence for a fly without implanted electrodes. The pattern of deviation and amplitude modulations in this fly was very similar to that in Fig. 3D. The kinematics of flies with and without electrodes were qualitatively indistinguishable within the range of differences seen among individuals. 

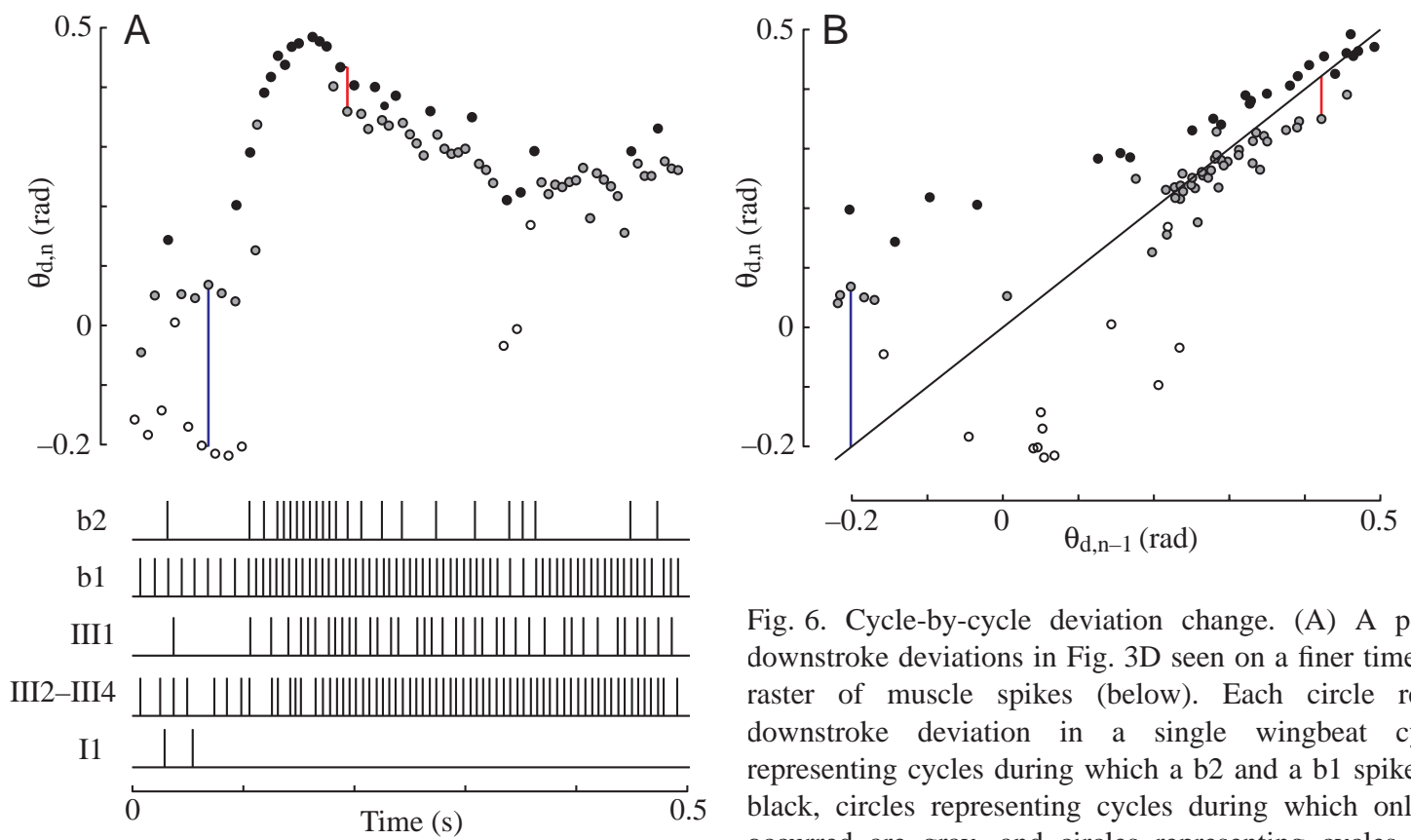

Fig. 6. Cycle-by-cycle deviation change. (A) A portion of the downstroke deviations in Fig. 3D seen on a finer time scale, with a raster of muscle spikes (below). Each circle represents the downstroke deviation in a single wingbeat cycle. Circles representing cycles during which a b2 and a b1 spike occurred are black, circles representing cycles during which only a b1 spike occurred are gray, and circles representing cycles during which neither a b2 nor a b1 spike occurred are white. One example of an increase in downstroke deviation, $\theta_{\mathrm{d}}$, from one cycle to the next is outlined in blue, and one example of a decrease in $\theta_{\mathrm{d}}$ from one cycle to the next is outlined in red. $\theta_{\mathrm{d}, \mathrm{n}}$ is the value of the deviation in stroke $n$ and $\theta_{\mathrm{d}, \mathrm{n}-1}$ is the deviation in stroke $n-1$. (B) Each of the downstroke deviation values in A plotted as a function of the value in the previous cycle. The diagonal line indicates $\theta_{\mathrm{d}, \mathrm{n}}=\theta_{\mathrm{d}, \mathrm{n}-1}$. Points above this line represent an increase in $\theta_{\mathrm{d}}$ from the previous to the current cycle, and points below this line represent a decrease. The corresponding cycle-to-cycle increase in $\theta_{\mathrm{d}}$ outlined in A is indicated here in blue. The corresponding decrease in $\theta_{\mathrm{d}}$ outlined in $\mathrm{A}$ is indicated here in red.

and b1 remained active. However, other muscles were concurrently active, making it difficult to isolate the effects of the basalare muscles from what may be combinatorial effects of the basalares with other muscle groups. For example, the spike rate of b1 and III2-III4 increased nearly simultaneously (Fig. 6A). Furthermore, spike rates in muscles b2 and III1 reached maximal values at nearly the same time (Fig. 3B), but diverged in spike rate thereafter (Fig. 6A).

As in many of our recordings, the modulations of $\theta_{\mathrm{d}}$ shown in Fig. 6A appeared to occur in a cumulative series of cycleby-cycle 'steps', with each basalare muscle spike eliciting a change relative to the prior baseline. Such features are expected because the mechanical and physiological properties of the steering muscles limit the relaxation that can occur from one cycle to the next. Data from single twitches in b1 indicate that rise times are of the order of a single wingbeat cycle, whereas relaxation times are approximately $2-3$ wingbeat cycles for $50 \%$ relaxation (Bergmann-Erb and Heide, 1990; Tu and Dickinson, 1994). For this reason, it is useful to compare the kinematic pattern of each cycle with that of the prior cycle. Fig. $6 \mathrm{~B}$ plots the relationship between the value of deviation in one stroke, $\theta_{\mathrm{d}, \mathrm{n}}$, and the value in the previous stroke, $\theta_{\mathrm{d}, \mathrm{n}-1}$. The line $\theta_{\mathrm{d}, \mathrm{n}}=\theta_{\mathrm{d}, \mathrm{n}-1}$ indicates the points where downstroke deviation remains constant. Points above this line represent an increase and points below this line represent a decrease in the value of $\theta_{d}$ from one stroke to the next. Two trends emerged from mapping patterns of basalare activity onto the cycle-bycycle changes in downstroke deviation (Fig. 6B). First, the variation between $\theta_{\mathrm{d}, \mathrm{n}}$ and $\theta_{\mathrm{d}, \mathrm{n}-1}$ differed among the three patterns of $b 1$ and $b 2$ activity. Second, the changes from $\theta_{\mathrm{d}, \mathrm{n}-1}$ to $\theta_{\mathrm{d}, \mathrm{n}}$ associated with each spiking pattern depended on the magnitude of the baseline.

Plotting all measured values of $\theta_{\mathrm{d}}$ from several video sequences in the same manner revealed the consistency of these observed trends (Fig. 7A) (Table 1). The points representing the three patterns of basalare activity clustered within three distinct regressions differing most in their offset along the $\theta_{\mathrm{d}, \mathrm{n}}$ axis. This clustering indicates that patterns of basalare muscle activity in each cycle strongly influence the change in $\theta_{\mathrm{d}}$ from one cycle to the next (Fig. 7B). Cycles in which both b1 and b2 fired (black circles) were correlated with the largest increments in $\theta_{\mathrm{d}}$, and the size of the change diminished as the level of the baseline increased (Fig. 7A,B). Cycles in which only b1 fired (gray circles) were correlated with somewhat smaller increases in $\theta_{\mathrm{d}}$ and showed a similar dependence on absolute baseline (Fig. 7A,B). Cycles in which neither muscle fired (white circles) were correlated with a decrease in $\theta_{\mathrm{d}}$, with the size of the decrease increasing as the magnitude of $\theta_{\mathrm{d}, \mathrm{n}-1}$ rose (Fig. 7A,B). Within each video sequence, the $y$ intercept of the ' $\mathrm{b} 2+\mathrm{b} 1$ ' spiking pattern regression (red line) was always the largest, that of the "b1 only' regression (green line) intermediate and that of the 'neither b1 nor b2' regression (blue line) the smallest (Fig. 7C) (Table 1). The difference among the three regressions probably reflects the synergistic interactions between $\mathrm{b} 1$ and $\mathrm{b} 2$ on the position of the basalare sclerite. During flight, the basalare 


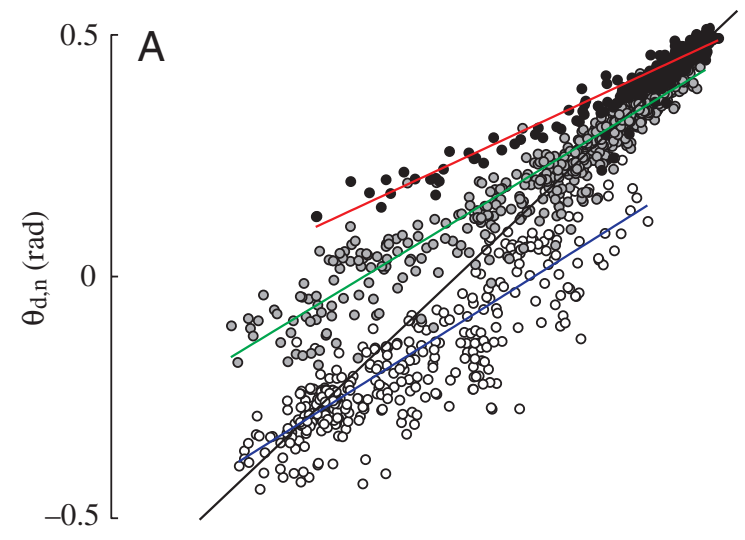

apodeme oscillates back and forth in phase with the stroke cycle (Tu, 1995). If b1 fires, the mean position of the basalare apodeme moves further anterior, causing a larger stroke deviation. If both $\mathrm{b} 1$ and $\mathrm{b} 2$ fire, the influence on the kinematics of the sclerite and the resultant increase in stroke deviation is greater. If neither motor neuron fires, the muscles relax, the mean position of the basalare apodeme moves posteriorly and stroke deviation diminishes.

The intercepts within spiking categories differed among sequences, especially within the 'b1 only' category (Fig. 7C) (Table 1). Much of this variation in the correlated effects of b1 and b2 spikes appeared to stem from differences in the specific timing of the spike within the wingbeat cycle, or spike phase, as will be discussed in the following section.

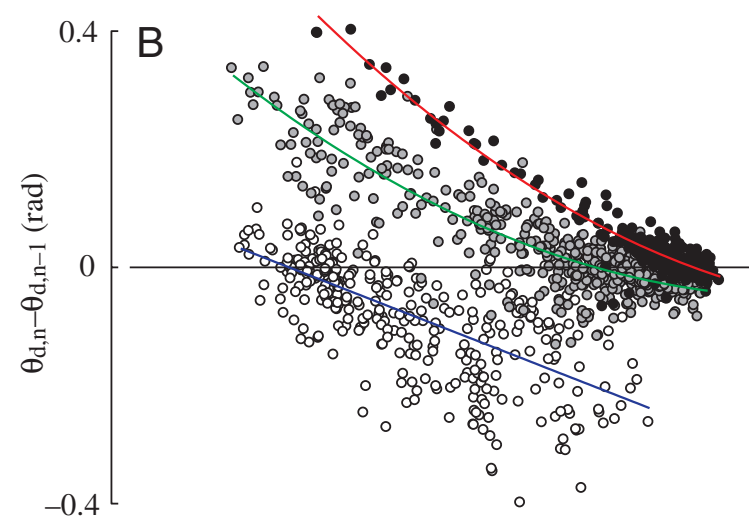

\section{Downstroke deviation and spike phase}

The specific timing of muscle spikes within the cycle is also known to be an important determinant of the mechanical output of the b1 (Tu and Dickinson, 1994, 1996) and possibly of other steering muscles. Consistent with these previous findings, much of the variation within the b1- and b2-related effects on $\theta_{\mathrm{d}}$ was associated with the specific phase of the spike occurrence, $\psi$. Fig. 8A maps the firing phase of $\mathrm{b} 1$ and $\mathrm{b} 2$ onto the regressions of Fig. 7A using pseudocolor. As with Fig. 7A, the position of each point relative to the line $\theta_{\mathrm{d}, \mathrm{n}}=\theta_{\mathrm{d}, \mathrm{n}-1}$ indicates whether downstroke deviation decreased, increased or stayed the same from one stroke to the next. For any given value of $\theta_{\mathrm{d}, \mathrm{n}-1}$, the firing phase of basalare spikes was well correlated with the direction and magnitude of change in $\theta_{\mathrm{d}}$ (Fig. 8A,B). Regressions through points representing similar b1 spike phases produced roughly parallel lines with intercepts that rose with decreasing $\psi$ (Fig. 8C). The points at which these lines intersect the line of unity $\left(\theta_{\mathrm{d}, \mathrm{n}}=\theta_{\mathrm{d}, \mathrm{n}-1}\right)$ represent the predicted maintained downstroke deviation were b1 to fire at wingbeat frequency with constant phase. Thus, by tonically adjusting firing phase, the fly could maintain different levels of downstroke deviation. The relationship between b1 spike phase and the point of intersection with the line of unity was consistent with our observation that, even when b1 fired at maximal frequency, the measured downstroke deviations could be low if b1 fired late in the cycle.

Overall, b1 and b2 spike rate and phase were robust

Fig. 7. Spike-type-dependent regressions of deviation change. (A) Each downstroke deviation measured in three sequences (1105 cycles) of oscillatory turning in a single individual plotted as a function of the downstroke deviation in the previous wingbeat cycle. The color code for circles is as in Fig. 6. The red line is a Model I regression through all black points, the green line is the regression through all gray points and the blue line is the regression through all white points. The diagonal black line indicates $\theta_{\mathrm{d}, \mathrm{n}}=\theta_{\mathrm{d}, \mathrm{n}-1} . \theta_{\mathrm{d}, \mathrm{n}}$ is the value of the deviation in stroke $n$ and $\theta_{\mathrm{d}, \mathrm{n}-1}$ is the deviation in stroke $n-1$. (B) Data points in A re-plotted to show the change in downstroke deviation from one cycle to the next as a function of the starting deviation. The red line is a second-order Model I regression through the black points, and the green line is a second-order Model I regression through the gray points. The blue line is a first-order regression through all white points. (C) Spike-type-dependent regressions as in A shown for eight individuals. within and across individuals. We were unable to find evidence of interactions of $b 1$ and $b 2$ with the other recorded muscles in determining downstroke deviation. However, the deviation values during cycles lacking b1 and b2 activity did vary within and across individuals (Fig. 7) (Table 1). A proportion of this variation was related to changes in the lower limits of $\theta_{\mathrm{d}}$, as will be discussed in the following section.

\section{Downstroke deviation and forward amplitude}

Although the wing hinge is complex, the fly is nevertheless limited in the way it can adjust its kinematics throughout the stroke. For example, maximum forward amplitude $\left(\phi_{\max }\right)$ was strongly correlated with downstroke deviation $\left(\theta_{\mathrm{d}}\right)$ in all 
Wing kinematics and steering muscle activity in Calliphora vicina 4221

Table 1. Moder regresion statitic for the regresions shown in Fig. $7 C$

\begin{tabular}{|c|c|c|c|c|c|c|c|c|}
\hline & \multicolumn{8}{|c|}{ Sequenice } \\
\hline & $\operatorname{mlt} 7^{*}$ & m1910 & minon & malo4 & m2410 & $\mathrm{n} 1102$ & $\mathrm{~m} 1400$ & nlone \\
\hline \multicolumn{9}{|c|}{$\bar{b}$} \\
\hline Int $=\mathrm{n} x=\mathrm{pt}$ & 0.27 & $0.10 \pi$ & 0.100 & 0.2 & 0.161 & 0.15 & 0.002 & 0.11 \\
\hline slope & 0.49 & 0.517 & 0.479 & 0.443 & $0 . \omega$ & 0.312 & 0.621 & $0 . \$ 1$ \\
\hline$M$ & 20 & $\theta$ & 115 & 95 & 6 & 41 & 75 & 91 \\
\hline $\mathrm{r}^{2}$ & 0.80 & 0.0 & $0 \leqslant 1$ & $0 . \pi 07$ & 0.86 & 0.867 & 0.61 & 0.00 \\
\hline $\mathbf{g}$ & 0.001 & 0.001 & 0.01 & 0.001 & 0.001 & 0.001 & 0.001 & 0.001 \\
\hline \multicolumn{9}{|l|}{ bl } \\
\hline Int $=-\pi=p t$ & 0.105 & 0,000 & $-0,004$ & 0.060 & $-0,04$ & $-0,00$ & -0.141 & $-0,002$ \\
\hline sloge & 0.84 & $0 . \theta$ & 0.74 & 0.70 & 0.6 & 0.60 & 0.841 & 0.0 \\
\hline$M$ & $\$ 1$ & 112 & 130 & 60 & 175 & 20 & 202 & 130 \\
\hline$r^{2}$ & 0.91 & 0.704 & 0,67 & 0.919 & $0.0 \%$ & 0.729 & 0.645 & 0.65 \\
\hline$g$ & 0.001 & 0.001 & 0.01 & 0.001 & 0.01 & 0.001 & 0.01 & 0.001 \\
\hline \multicolumn{9}{|c|}{ Heidnerbl norto } \\
\hline In & -0.112 & $-0,060$ & -0.142 & -0.11 & -0.161 & $-0,57$ & $-0,0$ & $-0,35$ \\
\hline slope & 0.60 & 0.50 & 0.712 & $0 . \$ 6$ & 0.843 & 0.192 & 0.200 & 0.003 \\
\hline$M$ & $3 \%$ & $1 \theta$ & 134 & 10 & 117 & $\pi$ & $\pi$ & 150 \\
\hline$r^{2}$ & 0.70 & 0.617 & 0.71 & $0 . \$ 2$ & 0.51 & 0.00 & 0.075 & 0.01 \\
\hline g & 0.001 & 001 & 001 & 0.001 & $0 . \infty 1$ & 0.01 & 0.05 & 1 \\
\hline
\end{tabular}

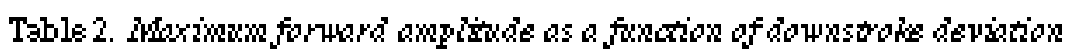

\begin{tabular}{|c|c|c|c|c|c|c|c|c|c|c|}
\hline & \multicolumn{10}{|c|}{ Sequence } \\
\hline & m1Tl1s & m171 & m1910 & mano & mon & mool & $m 2410$ & mllon & m1400 & nitor \\
\hline Int $=n x=p t$ & 0.791 & 1.0471 & $0.4 \hat{8}$ & 0.999 & 0.928 & $0 . \pi 9$ & 0.08 & 1.079 & 0.810 & 1.000 \\
\hline slope & 0.6 & $1.2 \% 7$ & 1.173 & 0.02 & 1.190 & $1 \omega \theta$ & 0.967 & $0 . \pi 2$ & 0,0 & 0.741 \\
\hline$N$ & $3 \pi$ & 36 & 307 & 364 & $3 \%$ & 346 & $3 \pi$ & 351 & 300 & $3 \pi$ \\
\hline$r^{2}$ & 0.6 & 0.002 & 0.756 & 0.885 & 0.005 & 0.914 & 0.84 & 0.756 & 0.80 & 0,00 \\
\hline$g$ & 0.001 & 0.001 & 0.001 & 0.001 & 0.001 & 0.001 & 0.001 & 0.001 & 0.001 & 0.001 \\
\hline
\end{tabular}

animals (Table 2). This correlation suggests that, because of a structural or mechanical linkage, when the basalare muscles elicit a change in downstroke deviation, there is a concomitant increase in forward amplitude. However, there exists enough variation in the correlation between downstroke deviation and forward amplitude to suggest that play in the mechanical linkages of the wing hinge permits additional muscles to modify the effects of the basalare muscles.

Within the visually induced oscillatory turning reactions, b1 and III2-III4 were nearly always simultaneously active, and it was therefore difficult to tease apart their independent contributions to wingbeat kinematics. For instance, in Fig. 9A, maximal firing rates in III2-III4 were associated with large amplitudes (blue) and low III2-III4 firing rates were associated with small amplitudes (red), but these changes may be due to the correlated activity of b1 with III2-III4. When the tethered flies were allowed to fly without the moving visual stimulus, III2-III4 tended to remain maximally active while the spike rate of b1 varied (Fig. 9B). Under these conditions, III2-III4 activity was not associated with any particular range of amplitudes. This suggests that only the basalares affect amplitude concomitantly with their effect on deviation and that the apparent correlation between III2-III4 activity and amplitude in Fig. 9A was indeed a result of its correlated activity with b1 rather than a real contribution to this kinematic parameter. However, by overlaying these data with respect to $\theta_{\mathrm{d}}$ (Fig. 9C, left), a subtle trend emerged. Maximal activity in III2-III4 resulted in a curvilinear relationship between amplitude and deviation (Fig. 9C, blue-green curve) that was consistently distinct from the relationship when III2-III4 was not active or firing at low frequency (Fig. 9C, red curve).

These two curves are different from each other in two ways. First, they differ in their relative offset along the $\phi_{\max }$-axis, indicating that, for a given deviation, the accompanying amplitude is larger when III2-III4 is firing maximally than when III2-III4 is firing submaximally. Therefore, while the basalares appear to control most of the variation along the $\theta_{\mathrm{d}^{-}}$ axis and concomitantly control $\phi_{\max }$ in part as described above, 

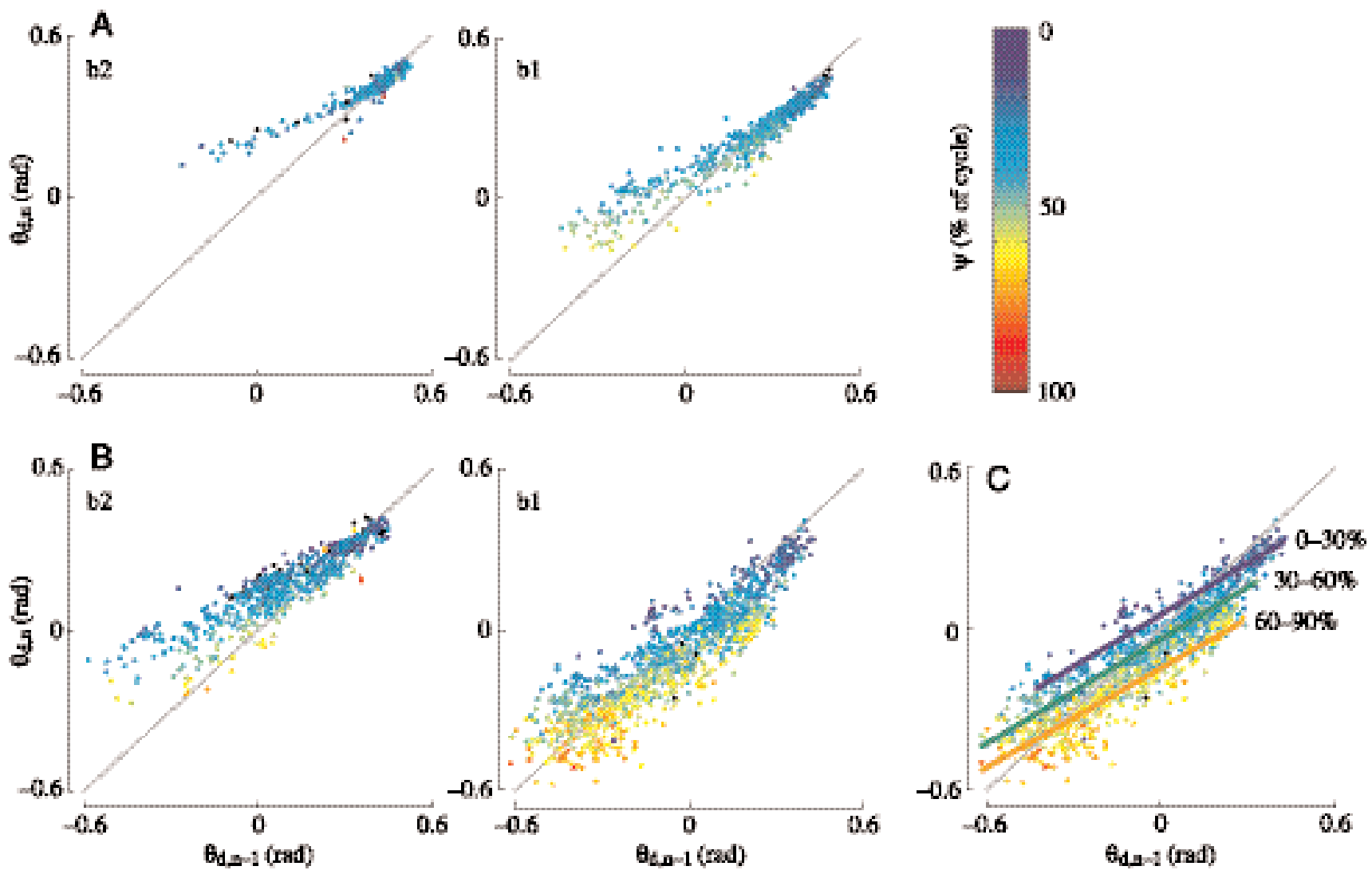

Fig. 8. Downstroke deviation in relation to shifts in firing phase. In all panels, the gray diagonal line represents $\theta_{\mathrm{n}}=\theta_{\mathrm{n}-1}$. $\theta_{\mathrm{d}, \mathrm{n}}$ is the value of the deviation in stroke $n$ and $\theta_{\mathrm{d}, \mathrm{n}-1}$ is the deviation in stroke $n-1$. Firing phase is denoted by color according to the color bar on the right, with blues representing the earliest phases and reds the latest. Black points indicate the occurrence of two spikes within one wingbeat cycle. (A) In the graph on the left, the black circles from Fig. 7A are re-plotted, with each point color-coded according to the firing phase of b2. In the graph on the right, the gray circles from Fig. 7A are replotted, with each point color-coded according to the firing phase of b1. (B) Composite graphs of downstroke deviations in seven sequences from seven individuals. Deviations from cycles in which a b2 and a b1 spike occurred are shown on the left, deviations from cycles in which only a b1 spike occurred are shown on the right, and all points are color-coded according to the firing phase of b2 or b1 as in A. (C) The b1-dependent deviations from B. Regressions through points representing deviation changes following spikes occurring during the first (0-30\%), second (30-60\%) or last third (60-90\%) of the wingbeat cycle produce three nearly parallel lines.

III2-III4 contributes to control of $\phi_{\max }$. Second, the two curves differ in their lower limits to deviation and amplitude. During low III2-III4 activity, downstroke deviations dipped no lower than approximately $-0.3 \mathrm{rad}$ and forward amplitudes to no lower than approximately $0.4 \mathrm{rad}$ (red curve; Fig. 9C). In contrast, when III2-III4 fired at wingbeat frequency, downstroke deviations fell as low as $-0.7 \mathrm{rad}$ and stroke amplitudes approached 0 rad (green curve; Fig. 9C). Similar relationships between III2-III4 activity and covariation of forward amplitude with downstroke deviation were seen in other individuals (Fig. 9D, left).

The deviation/amplitude relationship corresponding to low III2-III4 activity (red curve) was also loosely associated with high I1 activity (Fig. 9C,D, right). Although I1 was not always active when forward amplitude varied with downstroke deviation as indicated by the red curve in Fig. 9C,D, high I1 activity was consistently correlated with the smallest downstroke deviations and forward amplitudes (black points in Fig. 9C,D). This lower plateau in amplitude and deviation was not the lowest achievable, as noted above.

\section{Discussion}

Using simultaneous high-speed video and multi-channel EMG recordings, we correlated kinematic variation in wing motion with the activity of a population of steering muscles. The variability of firing patterns within and among sequences made it possible to quantify the context-dependence of the correlated effects of muscle activity. Because of the complexity of the neuromuscular and musculoskeletal components of the wing hinge, a number of factors may contribute to the way in which spike patterns in motor neurons are converted to wing motion. We found evidence for three distinct but interrelated features of the neuromechanical linkage of the flight system. First, correlations between basalare spike arrival times and kinematic changes suggest that certain dynamic properties of steering muscles are important in determining wing kinematics. Second, the action of the basalare muscles was dependent on the activity of other steering muscles. Lastly, steering muscles may alter wing motion qualitatively by toggling the system between different gears. 

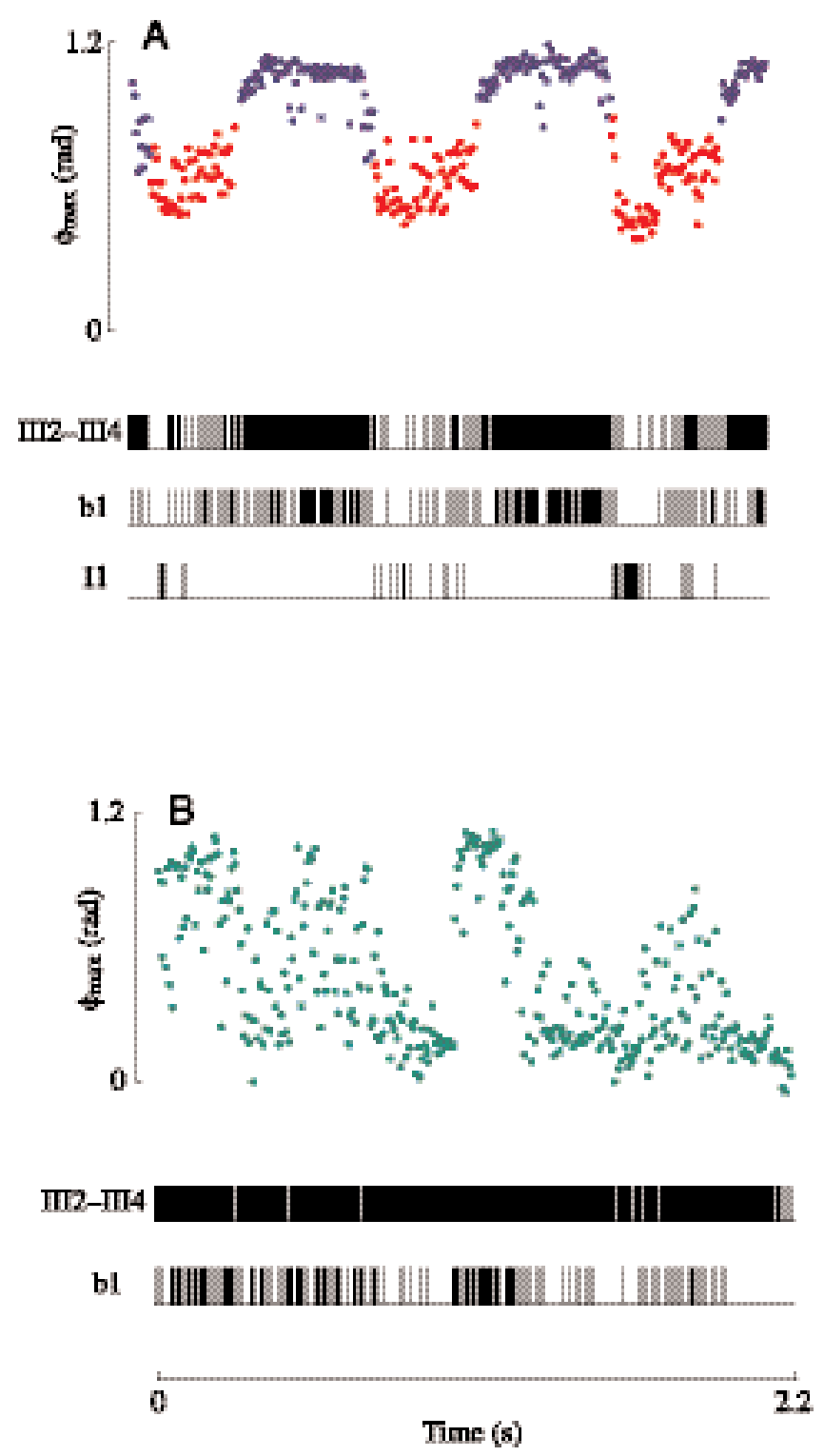
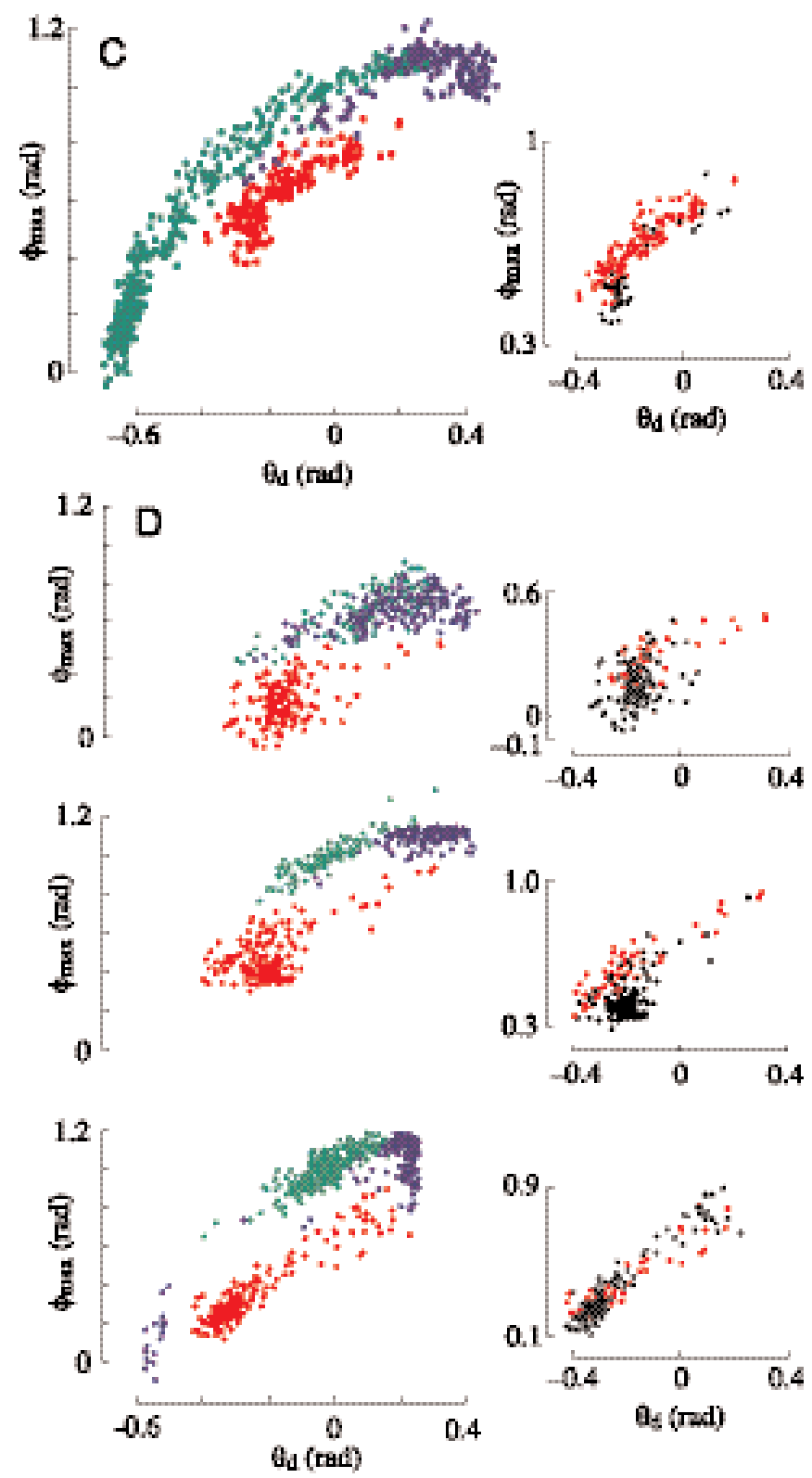

Fig. 9. Patterns of maximum forward amplitude modulations. (A) Maximum forward amplitude ( $\left.\phi_{\max }\right)$ modulations during one sequence of visually induced oscillatory turning. Spiking in III2-III4 increased and decreased in frequency following a bursting pattern. For simplicity, III2-III4 was defined as 'ON' when spikes occurred at or above wingbeat frequency. The blue points represent the amplitudes measured while III2-III4 fired maximally, and the red points represent the amplitudes measured while III2-III4 did not fire or fired submaximally. Raster plots of the concomitant spike occurrences in b1, III2-III4 and I1 are shown at the bottom. (B) Forward amplitude modulations in the same individual during a flight sequence with no moving visual stimulus. III2-III4 fired at maximal frequency through the entire sequence. All points are colored green to represent constant III2-III4 activity. Raster plots of b1 and III2-III4 are shown as in A. (C) Forward amplitudes from the sequences in $\mathrm{A}$ and $\mathrm{B}$ plotted as a function of downstroke deviation. The blue and red points represent the blue and red points in A, and the green points represent all points in B. The red points are re-plotted on the right, with black points indicating cycles during which a I1 spike occurred. (D) Relationship between downstroke deviation $\left(\theta_{\mathrm{d}}\right)$ and forward amplitude in three additional individuals. As in C, blue points represent the two kinematic parameters measured while III2-III4 fired maximally, red points represent parameters measured while III2-III4 fired submaximally, and green points represent parameters measured during a continuous sequence of maximal III2-III4 activity. On the right, the red points are re-plotted, as in C.

\section{Dynamic properties of the basalare muscles}

Of the various activation parameters that can influence force development in a muscle, the rate and phase of activation are typically most potent (see Josephson, 1985). In the blowfly
Calliphora vicina, the muscle properties of one steering muscle, b1, have been particularly well studied (Bergmann-Erb and Heide, 1990; Tu and Dickinson, 1994) and are linked to the control of wingbeat kinematics (Tu and Dickinson, 1996). 
For muscle b1, phase shifts have emerged as the dominant control parameter used to adjust work output (Heide, 1983; Tu and Dickinson, 1994, 1996). In contrast, the basalare muscle b2 appears to exert its influence on wing kinematics primarily through changes in rate, with firing phase being relatively constant within bursts of activity (Heide, 1983; Tu and Dickinson, 1996). However, given the variety of muscle activation patterns we identified in the present study, the distinctions between the control mechanisms of b1 and b2 become less clear. Activation of both these muscles can occur at a multitude of spike rates and phases (Figs 3, 4). Over the range of these firing patterns, the correlations between b1 versus b2 spikes and the associated kinematic parameters, such as downstroke deviation and forward amplitude, diminish (Tu and Dickinson, 1996). We found that, by correlating b1 and b2 spike occurrences with cycle-to-cycle changes in downstroke deviation (Figs 7, 8), these two muscles could account for a large proportion of the observed variation in wing trajectory during visually induced steering reactions.

Our finding that the changes in downstroke deviation are dependent on the absolute level of stroke deviation suggests at least two similar hypotheses for the way muscle properties influence the modulation of kinematics. First, because of intrinsic physiological properties, the size of each twitch might be dependent on the rate of activation (Aidley, 1985). Such effects have been demonstrated under isometric conditions for muscle b1 (Tu and Dickinson, 1994). Second, because the muscles operate within a complex system of skeletal attachments, the mechanical advantage, and thus the net effect on the wing, might change with the baseline level of activity. These hypotheses are not mutually exclusive. Either mechanism, a physiological rate-dependence or a changing mechanical advantage, could explain the diminished influence of muscle spikes at elevated baselines. Further, because the basalare muscles are attached to a common sclerite, the activity of any one muscle could alter either the length-dependent physiological properties or the mechanical advantage of the others. Our data from two of the muscles, b1 and b2, suggest that both muscles contribute to the summation process controlling downstroke deviation. Through these complex interactions, the sequence (Fig. 7) and the timing (Fig. 8) of spikes in just a few motor neurons exert a remarkably flexible control over motor output.

The cycle-by-cycle variation in downstroke deviation during cycles in which neither b1 nor b2 fired was relatively high (Fig. 7) (Table 1). This suggests that additional factors contribute to variation in this kinematic parameter, most notably the unrecorded activity of the remaining basalare muscle b3, which is positioned to act as an antagonist to b1 and b2, and the activity of muscles attached to the other wing sclerites.

\section{Interaction between steering muscle groups}

The steering muscles of flies have typically been grouped functionally according to the sclerites to which they are attached (see Wisser and Nachtigall, 1984). The functions assigned to each of these muscle groups have been that of either increasing or decreasing specific kinematic parameters, such as stroke amplitude averaged over many wingbeats. However, our findings suggest that this form of categorization needs to be modified for the real-time case. For example, as described in the previous section, the basalares can increase or decrease downstroke deviation and forward amplitude depending on the prior baseline. Further, because the basalares are probably not the only contributors to modulations in forward amplitude, the classification scheme must be modified to include the combined effects of concomitantly active steering muscles.

Consistent with the idea that combined activity of multiple muscles contributes to the conversions linking muscle spikes to kinematics, we found that the correlation between basalare activity and forward amplitude depended on the concomitant activity of muscles III2-III4. As shown in Fig. 9C,D and summarized in Fig. 10, the forward amplitude accompanying a given downstroke deviation is larger while III2-III4 is active (blue-green curve) than when it is not (red curve). The relatively small forward amplitudes accompanying each downstroke deviation are associated with the activity of I1 to some degree (Fig. 9C,D), but may also be due to the unrecorded activity of $\mathrm{I} 2$. Therefore, the manner in which the basalares control forward amplitude appears to depend on the activity of the muscles attached to both sclerites I and III.

The differences we found in the relationship between deviation and amplitude are consistent with some previous observations of wing hinge configuration in relation to wing kinematics. The presence of a grooved structure beneath the wing articulation, the pleural wing process (PWP), and a projection at the base of the wing, the radial stop (RS), which is shaped and positioned to fit into the grooves of the PWP, has inspired several hypotheses concerning their function in dipteran flight control (for a review, see Nalbach, 1989). Nalbach's (1989) observations of these structures during tethered flight supported the hypothesis that the PWP can act as a wing stop during the latter half of the downstroke, as the RS engages in one of the grooves of the PWP. In addition, she inferred that the placement of the RS relative to the PWP as the wing descended was under neuromuscular control because these structures appeared to operate in several distinct modes. The RS could pass in front of the PWP (mode 3 ), engage in the anterior groove of the PWP (mode 2), engage in the posterior groove of the PWP (mode 1) or pass behind the PWP (mode 0) during the downstroke. The correlation she found between operating mode and downstroke amplitude suggested mode selection as one possible mechanism of amplitude control. However, she also found that downstroke amplitude could be decreased with or without the contact of the RS with PWP.

Nalbach (1989) attempted to correlate these kinematic changes with the activity of basalar muscles by observing the deflection of the basalar sclerite. On the basis of sclerite motion, contraction of b1 and b2 correlated with large stroke amplitudes and relaxation correlated with small stroke amplitudes, but motion of the basalare sclerite did not always coincide with mode switching. Motion of axillare 3 (pterale III), however, was closely correlated with mode switching. 
Wing kinematics and steering muscle activity in Calliphora vicina 4225

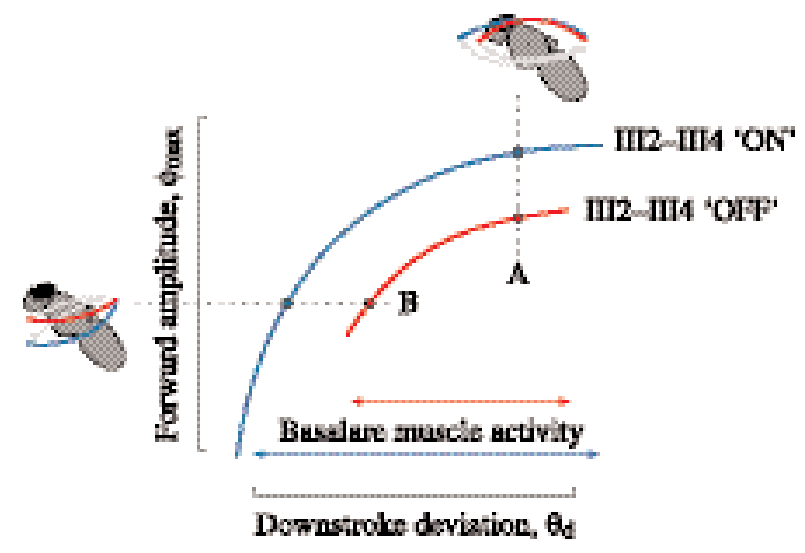

Fig. 10. Diagram of the relationship between downstroke deviation and forward amplitude when III2-III4 is active (blue) and when III2-III4 is not active (red). (A) Difference in forward amplitude under these two conditions given the same deviation value. A representative downstroke trajectory corresponding to each point is shown above. (B) Difference in downstroke deviation given the same amplitude value. A representative downstroke trajectory corresponding to each point is shown on the left.

Although Nalbach (1989) did not speculate about the mechanism by which the muscles of axillare 3 might participate in the control of mode selection, she hypothesized that the muscles of axillare 1 (pterale I) might pull the wing medially so that the RS contacts the PWP.

Our results from recordings of muscle activity are in accordance with Nalbach's (1989) inferences from sclerite motion. The spike activity of b1 and b2 was strongly correlated with forward amplitude (Table 2). In our analysis, we also found that low-frequency b1 and b2 activity combined with elevated activity in I1 was correlated with relatively large lower limits to downstroke deviation and forward amplitude compared with the limits achieved when III2-III4 was active (Fig. 9C,D). This kinematic limit associated with I1 activity might be due to a mechanical obstruction such as that caused by contact of the RS with the PWP during the downstroke, whereas the activity of III2-III4 might keep the two structures from engaging. In this case, the muscles of sclerites I and III appear to control 'mode-switching', as proposed by Nalbach (1989), while the basalares effect changes in forward amplitude within modes.

Our results also confirm the proposal of Heide (1971b) that the muscles of pterale III support the basalare muscles in regulating the stroke amplitude. However, our analysis considered only the 'ON' or 'OFF' effects of III2-III4 firing, and further contributions of the spike rate, firing phase and muscle recruitment remain to be quantified. The second role of the pterale III muscles postulated by Heide (1971b), regulating the angle of attack, cannot be tested without reconstructing the three-dimensional kinematics of the wing. Because of its relatively phasic firing pattern under the current experimental conditions and its large size among steering muscles, we would expect each III1 spike to cause detectable changes in wing kinematics. The fact that III1 firing did not correlate with changes in wingtip trajectory suggests that its action may regulate some unmeasured kinematic parameters such as angle of attack.

\section{Lessons from free flight}

Although any extrapolation from the kinematics of one wing of a tethered fly must be made with caution, we can begin to address the implications for the neuromechanical control of free flight maneuvers. In his study on Musca domestica, Wagner (1986b) postulated that some of the delays in flight correction may be due to 'summation effects in the muscle system'. The additive effects of the basalare muscles we observed indicate that cycle-by-cycle kinematic changes are indeed limited, and several cycles of repetitive activity are required to elicit substantial changes in kinematic parameters such as stroke deviation and amplitude. Further, both Wagner (1986a) and Schilstra and van Hateren (1999) observed that, while flies have the ability to alter yaw, pitch and roll independently, these directional parameters vary together in a characteristic manner during the most common turning behaviors. Blondeau (1981) also described similar results for directional forces measured in tethered Calliphora erythrocephala. On the basis of these findings, wingbeat kinematics should similarly co-vary in a characteristic manner, while occasionally displaying a decoupling as observed in behavioral studies (Nachtigall and Roth, 1983). In accordance with this expectation, we have observed one example in which downstroke deviation $\left(\theta_{\mathrm{d}}\right)$ and forward amplitude $\left(\phi_{\max }\right)$, while typically covariant, were decoupled depending on differential III2-III4 activity. Further examination of wingbeat kinematics may reveal the partial decoupling of other kinematic parameters.

In summary, we found that the role of any single muscle cannot be considered in temporal or spatial isolation either from its prior activity or from the action of other steering muscles. Our correlational analysis identified some of the muscular and structural features that may influence the context-dependent effects of muscle activity on wingbeat kinematics. The causal relationships remain hypotheses that have yet to be tested and will require experiments involving the ablation and stimulation of individual muscles. Further, a larger data set is required to increase the range of observed wing kinematics and to identify the influence of other steering muscles. Most importantly, it will be necessary in future experiments to measure the instantaneous angle of attack of the wing during the types of wingbeat modulation observed in the present study. Recent studies of the kinematics and aerodynamics of Drosophila melanogaster have quantified the importance of subtle changes in angle of attack and rotation timing on force output (Dickinson et al., 1999; Sane and Dickinson, 2001). Therefore, extending the analysis to threedimensional wing kinematics will make it possible to explore the aerodynamic consequences of steering muscle activity and to identify other kinematic parameters that may be influenced by these muscles. With such continued studies, we may begin to decode motor patterns in terms of their interactions with the musculoskeletal system and the surrounding medium. 
We would like to thank S. L. Lehman and F. E. Theunissen for helpful discussions during the early stages of this research. The manuscript was greatly improved by critical comments from S. P. Sane, M. A. Frye, S. D. Gordon and two anonymous referees. This work was supported by grants from the NSF (IBN-9723424) and Defense Advanced Research Projects Agency and Office of Naval Research (FDN0001499-1-0892) to M.H.D. and a U.C. Berkeley Chancellor's Opportunity Fellowship to C.N.B.

\section{References}

Aidley, D. J. (1985). Muscular contraction. In Nervous System: Structure and Motor Function, vol. 5 (ed. G. A. Kerkut and L. I. Gilbert), pp. 407-437. Oxford: Permagon Press.

Bergmann-Erb, D. and Heide, G. (1990). Kontraktionsmodus direkter Flugsteuermuskeln von Calliphora. In Proceedings of the 18th Göttingen Neurobiology Conference (ed. N. Elsner and G. Roth), p. 41. Stuttgart: Thieme Verlag.

Blondeau, J. (1981). Aerodynamic capabilities of flies, as revealed by a new technique. J. Exp. Biol. 92, 155-163.

Dawson, J. W., Dawson-Scully, K., Robert, D. and Robertson, R. M. (1997). Forewing asymmetries during auditory avoidance in flying locusts. J. Exp. Biol. 200, 2323-2335.

Dickinson, M. H., Farley, C. T., Full, R. J., Koehl, M. A. R., Kram, R. and Lehman, S. (2000). How animals move: An integrative view. Science 288, 100-106.

Dickinson, M., Lehmann, F.-O. and Sane, S. P. (1999). Wing rotation and the aerodynamic basis of insect flight. Science 284, 1954-1960.

Dickinson, M. H. and Tu, M. S. (1997). The function of Dipteran flight muscle. Comp. Biochem. Physiol. 116A, 223-238.

Egelhaaf, M. (1989). Visual afferences to flight steering muscles controlling optomotor responses of the fly. J. Comp. Physiol. A 165, 719-730.

Fischer, H. and Kutsch, W. (1999). Timing of elevator muscle activity during climbing in free locust flight. J. Exp. Biol. 202, 3575-3586.

Götz, K. G. (1983). Bewegungssehen and Flugsteuerung bei der Fliege Drosophila. In BIONA-Report 2: Insect Flight II (ed. W. Nachtigall), pp 21-34. Stuttgart: Fischer.

Heide, G. (1968). Flugsteuerung durch nicht-fibrilläre Flugmuskeln bei der Schmeißfliege Calliphora. Z. Vergl. Physiol. 59, 456-460.

Heide, G. (1971a). Die Funktion der nicht-fibrillären Flugmuskeln von Calliphora. Teil I. Lage, Insertionsstellen und Innervierungsmuster der Muskelin. Zool. Jb. Physiol. 76, 87-98.

Heide, G. (1971b). Die Funktion der nicht-fibrillären Flugmuskeln von Calliphora. Teil II. Muskuläre Mechanismen der Flugsteuerung und ihre nervöse Kontrolle. Zool. Jb. Physiol. 76, 99-137.

Heide, G. (1975). Properties of a motor output system involved in the optomotor response in flies. Biol. Cybernetics 20, 99-112.

Heide, G. (1983). Neural mechanisms of flight control in Diptera. In BIONAReport 2: Insect Flight II (ed. W. Nachtigall), pp. 35-52. Stuttgart: Fischer.

Heide, G. and Götz, K. G. (1996). Optomotor control of course and altitude in Drosophila is correlated with distinct activities of at least three pairs of steering muscles. J. Exp. Biol. 199, 1711-1726.

Hooper, S. L. and Weaver, A. L. (2000). Motor neuron activity is often insufficient to predict motor response. Curr. Opin. Neurobiol. 10, 676-682.

Josephson, R. K. (1985). Mechanical power output from striated muscle during cyclic contraction. J. Exp. Biol. 114, 493-512.

Kammer, A. E. (1971). The motor output during turning flight in a hawkmoth, Manduca sexta. J. Insect Physiol. 17, 1073-1086.

Lehmann, F.-O. (1994). Aerodynamische, kinematische und elektrophysiologische Aspekte der Flugkrafterzeugung und Flugkraftsteuerung bei der Taufliege Drosophila melanogaster. Thesis, Eberhad-Karls-Universität, Tübingen.

Lehmann, F.-O. and Götz, K. G. (1996). Activation phase ensures kinematic efficacy in flight-steering muscles of Drosophila melanogaster. J. Comp. Physiol. A 179, 311-322.

Miyan, J. A. and Ewing, A. W. (1985). How Diptera move their wings: a reexamination of the wing base articulation and muscle systems concerned with flight. Phil. Trans. R. Soc. Lond. B 311, 271-302.
Möhl, B. (1985). The role of proprioception in locust flight control. I. Asymmetry and coupling within the time pattern of motor units. J. Comp. Physiol. A 156, 93-101.

Möhl, B. and Zarnack, W. (1977). Activity of the direct downstroke flight muscles of Locusta migratoria (L.) during steering behaviour in flight. II. Dynamics of the time shift and changes in the burst length. J. Comp. Physiol. 118, 235-247.

Nachtigall, W. (1966). Die Kinematik der Schlagflügelbewegungen von Dipteren. Methodische und Analytische Grundlagen zur Biophysik des Insektenflugs. Z. Vergl. Physiol. 52, 155-211.

Nachtigall, W. and Roth, W. (1983). Correlations between stationary measurable parameters of wing movement and aerodynamic force productin in the blowfly (Calliphora vicina R.-D.). J. Comp. Physiol. 150, 251-260.

Nachtigall, W. and Wilson, D. M. (1967). Neuro-muscular control of dipteran flight. J. Exp. Biol. 47, 77-97.

Nalbach, G. (1989). The gear change mechanism of the blowfly (Calliphora erythrocephala) in tethered flight. J. Comp. Physiol. A 165, 321-331.

Pearson, K. G. and Wolf, H. (1987). Comparison of motor patterns in the intact and deafferented flight of the locust. I. Electromyographic analysis. J. Comp. Physiol. A 160, 259-268.

Rieke, F., Warland, D., Steveninck, R. D. R. V. and Bialeck, W. (1997). Spikes: Exploring the Neural Code. Cambridge: MIT Press.

Sane, S. P. and Dickinson, M. H. (2001). The control of flight force by a flapping wing: lift and drag production by a flapping wing. J. Exp. Biol. 204, 2607-2626.

Schilstra, C. and van Hateren, J. H. (1999). Blowfly flight and optic flow. I. Thorax kinematics and flight dynamics. J. Exp. Biol. 202, $1481-1490$

Schmidt, J. and Zarnack, W. (1987). The motor pattern of locusts during visually induced rolling in long-term flight. Biol. Cybernetics 56, 397-410.

Thuring, D. A. (1986). Variability of motor output during flight steering in locusts. J. Comp. Physiol. A 158, 653-664.

Tu, M. S. (1995). Physiological and biomechanical analysis of flight control by two specialized steering muscles in the blowfly, Calliphora vicina. Thesis, University of Chicago.

Tu, M. S. and Dickinson, M. H. (1994). Modulation of negative work output from a steering muscle of the blowfly Calliphora vicina. J. Exp. Biol. 192, 207-224.

Tu, M. S. and Dickinson, M. H. (1996). The control of wing kinematics by two steering muscles of the blowfly, Calliphora vicina. J. Comp. Physiol. A 178, 813-830.

Wagner, H. (1986a). Flight performance and visual control of flight of the free-flying housefly (Musca domestica L.). I. Organization of the flight motor. Phil. Trans. R. Soc. Lond. B 312, 527-551.

Wagner, H. (1986b). Flight performance and visual control of flight of the free-flying housefly (Musca domestica L.). II. Pursuit of targets. Phil. Trans. R. Soc. Lond. B 312, 553-579.

Waldman, B. and Zarnack, W. (1988). Forewing movements and motor activity during roll manoeuvers in flying desert locusts. Biol. Cybernetics 59, 325-335.

Wendler, G. (1974). The influence of proprioceptive feedback on locust flight co-ordination. J. Comp. Physiol. 88, 173-200.

Wendler, G., Muller, M. and Dombrowski, U. (1993). The activity of pleurodorsal muscles during flight and at rest in the moth Manduca sexta (L.). J. Comp. Physiol. A 173, 65-75.

Wilson, D. M. and Weis-Fogh, T. (1962). Patterned activity of co-ordinated motor units, studied in flying locusts. J. Exp. Biol. 39, 643-667.

Wisser, A. (1987). Mechanisms of wing rotating regulation in Calliphora erythrocephala (Insecta, Diptera). Zoomorphol. 106, 261-268.

Wisser, A. and Nachtigall, W. (1984). Functional-morphological investigations on the flight muscles and their insertion points in the blowfly Calliphora erythrocephala (Insecta, Diptera). Zoomorphol. 104, 188-195.

Wootton, R. J. (1981). Support and deformability in insect wings. J. Zool., Lond. 193, 447-468.

Young, D. (1989). Nerve Cells and Animal Behavior. New York: Cambridge University Press.

Zarnack, W. (1988). The effect of forewing depressor activity on wing movement during locust flight. Biol. Cybernetics 59, 55-70.

Zarnack, W. and Möhl, B. (1977). Activity of the direct downstroke flight muscles of Locusta migratoria (L.) during steering behaviour in flight. I. Patterns of time shift. J. Comp. Physiol. 118, 215-233. 